\title{
Jung and the Neurobiology of the Creative Unconscious
}

\author{
Jonathan Erickson
}

\begin{abstract}
The Jungian theories of creativity, like much of Jungian psychology, emphasize the relationship between the conscious mind and the unconscious. This paper explores and elucidates the intriguing parallels between this Jungian framework and recent models that have emerged in the neuroscience of creativity.
\end{abstract}

Keywords: Jung, creativity, neurobiology, neuroscience, unconscious, flow

\section{Introduction}

Creativity is, and perhaps will always be, a great mystery. Though explanations will be given and measurements will be made, the simple phenomenon of something new coming into being is at heart akin to the miracle of life itself. Some may be content with technical explanations and strictly materialist accounts, but for many more, the glimmer of mystery remains.

Jung's psychology, built largely around the concept of the unconscious, keeps the doorway at least partially open to such mysteries. The unconscious is by definition, after all, that which we do not really have much awareness of. It is the great unknown within. As Jung once beautifully put it:

Consciousness, no matter how extensive it may be, must always remain the smaller circle within the greater circle of the unconscious, an island surrounded by the sea; and, like the sea itself, the unconscious yields an endless and self-replenishing abundance of living creatures, a wealth beyond fathoming. (1946/1954, p. 178, para. 366)

The language here is hardly in reference to a bleak existential void of unknowing. In Jung's conception, this void is filled with possibilities and potentials, a psychological cornucopia waiting to be discovered. Like so much else in his psychology, Jung's accounts of creativity were based in this ongoing discourse between the conscious mind and unconscious within.

On the other side of the spectrum, a tension exists between strictly materialist accounts of humanity and the mysteries of the vast unknown, a tension often on display in those branches of neuroscience that attempt to find neurobiological accounts of the essential aspects of the human psyche. Prominent among such endeavors are the attempts to produce a neurobiological model of creativity. Both creativity studies and neuroscience have undergone tremendous growth in recent decades, and a great number of theories abound to try to capture and explain the complex and perplexing phenomena of the human act of creation.

Rather than laud one approach over the other, this paper will explore and outline the parallels between Jung's conception of the creative unconscious and some recent neurobiological models for human creativity. These frameworks are not identical, nor 
should they be. But there is a surprising degree of common ground. By elucidating these common connections, both fields of study can be enriched and perhaps even come to find a deepened respect for one another.

\section{Jung, Creativity, and the Unconscious}

For Jung, creativity and the unconscious were inextricably linked. "New thoughts and creative ideas can appear which have never been conscious before. They grow up from the dark depths like a lotus, and they form an important part of the subliminal psyche" (1961/1976, p.198, para. 449). The metaphor of the lotus speaks of something beautiful arising out of the dark unknown depths of the psyche, notably not something that the conscious mind is doing or making happen. Rather the conscious mind observes, and is perhaps surprised, to see something meaningful arise from within what previously did not seem to exist. Creativity, in this sense, is not the work of the conscious ego, though ultimately it may well be for the conscious ego to decide what to do with the creative gifts from below.

This idea that creativity is something beyond, or more than, the rational mind appears several times throughout Jung's works. "The creation of something new is not accomplished by the intellect but by the play instinct acting from inner necessity. The creative mind plays with the objects it loves" (1921/1971a, p.123, para. 197). Again, there is strong sense that creativity exists beyond the grasp of mere intellectualism and instead taps into something deeper, perhaps even primal. At the same time, Jung introduces the concept of playfulness as equally essential, further rebuffing the notion that creativity can be an entirely dry, analytical affair. "Without this playing with fantasy no creative work has ever yet come to birth. The debt we owe to the play of the imagination is incalculable" (1921/1971b, p. 63, para. 93). Fantasy and imagination become the realm where new possibilities can emerge. As long as things remain completely rigid and fixed, there is no room for something new to come into existence. Playfullness requires flexibility and implies a capacity for holding things and ideas lightly. At the same time, the language here still implies some distance between the observing ego and the imagination. In the above quotation, imagination is not something that we do; rather imagination is something we owe a debt to, as though it were a third party generously providing us with the raw materials for our creative work.

Jung often alluded to a real sense of wisdom in the unconscious - a deep capacity for resolution, growth, and healing that naturally unfolds as we get more fully in touch with ourselves. In working with dreams, for example, he would sometimes encourage his patients to engage with the material through some kind of artistic expression as a means of discerning deeper insight. "Often the hands will solve a mystery that the intellect has struggled with in vain" (1957/1969, p. 86. para. 180). Here again is the theme of a certain amount of surrender to an unconscious creative process, allowing it to express itself without too much conscious analysis or interference. Jung named his psychotherapy technique analytical psychology, so we can presume that at various points a more analytical process would come into play. But in the moment of creative art-making as a means to engage further with the unconscious, just the opposite is called for: a willingness to set aside rational analysis and allow something deeper to flow through. 
So much of Jungian psychology proceeds by bringing the conscious mind into contact with the unconscious and even more so by facilitating a "mutual penetration of the conscious and unconscious" (1934/1954, p. 152, para. 327). It is a conjunction in which both parties are vital. Without a stabilizing rational ego to contain the conscious mind, encounters with the unconscious would be tantamount to a descent into madness. But without access to the depths of the unconscious, the conscious mind is a stranger to itself, inexorably set in its ways. The point is not to promote one over the other but rather to find a balance, a dialogue, and ultimately some degree of integration. From this inner alchemy, Jung believed, a genuine process of psychological transformation is possible.

This meeting between consciousness and the unconscious mind is so central to Jung's psychology that it is difficult to discuss his understanding of creativity without it. And as this paper will demonstrate, his concept of the necessary balance between consciousness and the unconscious holds a surprising number of parallels to contemporary accounts of creativity in the neurosciences.

\section{Contemporary Theories of Creativity}

The interdisciplinary field of creativity studies has experienced tremendous period of growth over the last two decades. From 1999 to 2009 alone, over 10,000 papers were published on creativity spanning multiple disciplines (Kaufman and Beghetto, 2009). I wrote my own bachelor's thesis on interdisciplinary creativity at UC Berkeley in 2004, and since that time the topic has never ceased to fascinate me personally or professionally.

The basic definition used most often used in contemporary creativity studies is that something can be considered creative if it is both novel and useful (Carson, 2010; Vartanian, Bristol, \& Kaufman, 2013). Thus, thinking up an idea or image that has no discernable use does not qualify as creative. Nor does making a thing that serves no purpose. Of course, the purpose and use of an idea or thing does not have to be pragmatic or material - it could be philosophical, educational, aesthetic, entertaining, and so on. A terrible idea or contraption can still make for a good joke. And by the same definition, an idea or object can be very useful without necessarily being creative - if it is simply an imitation of something that came before. Part of the fascination of creativity is that it implies a process whereby something emerges from nothing; a new thing now exists because of the creative process, something that had not existed in quite that form beforehand.

Some definitions of creativity are quite stringent. For example, Mihaly Csikszentmihalyi's (1996) landmark study on highly creative individuals required that all participants had made a major contribution to their field. Thus, any instances of personally meaningful creativity or creative projects that only transform the life of an individual or community were not considered. Csikszentmihalyi was in this case studying what is sometimes called extraordinary creativity, the kind that is associated with great artists, scientific geniuses, and masters of business and politics. But contemporary discourse has come to consider a much broader understanding. In addition to extraordinary creativity, Nancy Andreason (2005) talks about the importance of ordinary creativity - the day-to-day creative thinking that we all employ over the regular course of our lives. Much research in neuroscience has been more concerned with the latter, 
particularly because it is not easy to measure extraordinary works of creativity by any clear objective standard, much less find an adequate sample size. In Wired to Create, Kaufman and Gregiore (2015) take a stand for creativity as a basic human birthright, something that we all have access to-though they acknowledge that some people certainly cultivate and develop their creative capacities more than others. Depending on how it is used, Creativity can be strengthened like the muscles of the body or be ignored and left to lie fallow like an empty field.

The classical theoretical model for creativity, still debated today, is usually attributed to either Helmholtz (1896) or Wallas (1926). This model holds that creative work takes place in four stages: 1) preparation, 2) incubation, 3) illumination, and 4) verification/elaboration. The preparation phase includes research and planning, whether learning the intricacies of a scientific problem or immersing oneself in life to find the raw materials for an artistic project. The second and third phases, incubation and illumination, are linked. Incubation occurs when we remove conscious attention from the project, allowing it to gestate for a time with a relaxed focus. Gestation, in theory, eventually leads to a moment of illumination, when new solutions, ideas, images, and stories emerge suddenly into consciousness, as if out of nowhere. The parallels to Jung's notion of the lotus arising from the depths should be obvious. Finally, in the fourth phase, these creative insights are either subjected to further verification and testing or are integrated into the work through a process of elaboration. The entire process is easily conceived in a cyclical manner, with ongoing iterations of preparation, incubation-illumination, and elaboration as the work continues.

There is ongoing debate as to the efficacy of this model, with some criticizing it for being too simplistic and not applicable to all creative processes. Nevertheless, it bears mentioning because elements of the classical model continue to show up in contemporary studies, as the following sections of this paper will show. One notable aspect of the classical model is that it implies a necessity of balance between conscious processes (preparation, elaboration) and unconscious processes (incubation-illumination), something that is born out both in Jungian theory and several modern neurobiological models of creativity.

For example, this notion of balance between conscious and unconscious is paralleled in Dietrich and Haider's (2017) neurobiological theory of deliberate and spontaneous creativity. Deliberate creativity refers to the process of consciously working on a problem or project through a combination or analytical discernment and rational planning and decision-making. Spontaneous creativity, on the other hand, refers to the generation of ideas, images, and solutions outside of deliberate conscious control. Dietrich and Haider insist that although these two types of creativity almost always work together, they are in themselves distinct neurobiological process and must be studied as such. A similar division is described by Gregoire and Kaufman (2015), who divide the brain's creative capacity into processes of generation and selection. In the generation phase, new ideas and images arise without being consciously controlled. The selection phase is where the conscious mind comes in, sorting through the ideas and images generated and working them into the creative process in a more deliberate and rational way. 


\section{Neurobiological Accounts of Conscious Creativity}

There has been considerable pushback in recent years against the idea that creativity can be discretely located in one brain region or another. Because a given creative process could draw on many different kinds of information and information processing, holistic or global accounts of creative brain function are proving increasingly popular. Kaufman and Gregoire (2015) declare from the outset that "The creative process draws on the whole brain" (p. xxvi). Pfenninger (2001) argues that creativity is among the highest of brain functions precisely because it involves the complex integration of so many other functions that might not directly engage each other during non-creative processing. Likewise, Carson (2010) writes that "there is, of course, no 'creativity' center in the brain ... creative cognition is a complex mental phenomenon that involves multiple sequential acts that utilize widespread circuits in the brain" (p. 45). Carson does, however, outline a map of multiple interacting brains systems vital to creativity, including some, such as centers associated with judgment, planning, motivation, and language processing, that are more typically associated with normative, non-creative cognition. Arne Dietrich (2007) has been especially critical of any search for a discrete or monolithic creativity center in the brain, commenting that "we might as well try to locate the neural centers for thinking" (p. 24).

Nevertheless, there are several extant streams of research and discourse over which areas and functions of the brain seem to make the greatest contribution to creative work. Among these, perhaps the most well known is the theory that the right hemisphere of the brain is the creative hemisphere, while the left brain is the analytical hemisphere. Like many popular theories and myths, this idea grows out of a kernel of truth, in that the right hemisphere does appear to be more specialized for holistic, global, and associative processing of the big picture, while the left hemisphere is more specialized for linear analysis and processing discrete particulars (Kaufman et al., 2010). The earliest evidence for this theory comes from the research of Roger Sperry (1974) into split-brain patientsindividuals who had had the corpus callosum connecting the right and left hemispheres severed, meaning that each hemisphere was essentially processing information independently of the other. Because, when tested, the right brain was observed to be better at holistic thinking, it was labeled as the more creative of the two.

Ultimately, this interpretation has not withstood the test of time. McGilchrist (2009) reviews evidence that individuals with the split-brain condition show diminished levels of imagination and creativity, and concludes "the integrated functioning of both hemispheres is needed for such activity" (p. 198). Likewise, after reviewing the recent evidence that the hemispheres are indeed specialized, Kaufman et al. (2010) nevertheless conclude that creativity requires "the sequential and interactive engagement of both hemispheres, an interdisciplinary engagement between two "experts" (p. 222). In other words, the right hemisphere needs the linear analytical specialization of the left in order to provide direction and discernment, while the left hemisphere needs the holistic specialization of the right to transcend its own limited analysis and effectively engage with the big picture. Again, balance and integration are essential.

A related body of theory is organized around the idea that creativity requires a disinhibition function in the brain, meaning that creative brain states tend to be naturally inhibited until the disinhibition function activates. In the late 20th century research by 
Colin Martindale found that creativity correlates with reduced activity in brain regions associated with the inhibition of "abnormal" behavior (Kaufman et al., 2010). In reference to the more poetic language of depth psychology, clear parallels emerge here to the idea of the ego necessarily giving up control, and to some extent surrendering, to the unconscious. For Freud, this might be a matter of the superego stepping back to allow the libidinal energies of the id to flow freely. But Jung expanded the notion of libido to encompass the wider range of human experiences, including the creative and spiritual, and this more expansive construct also maps relatively well onto the current evidence for the disinhibition hypothesis.

More recent studies into disinhibition have kept the theory alive. Radel et al. (2015) found evidence that idea generation increases as inhibition decreases. R.E. Jung and Haier (2013) looked at frontotemporal dementia and brain lesion studies in relation to creativity and found that "lower brain integrity within left hemisphere brain structuresparticularly left anterior temporal and inferior parietal lobes-serves to "disinhibit" other brain regions associated with increased novelty generation" (p. 243). Incidentally, this finding does inadvertently add fuel to the fire with regards to viewing the analytical left brain as a potential antagonist to creative output. R.E. Jung and Haier ultimately interpret their evidence to argue for "a decidedly left-lateralized, frontosubcortical, and disinhibitory network of brain regions underlying creative cognition" (p. 244). In a similar study looking at neurodegenerative disorders, Vishkontas \& Miller (2013) concluded that "diminished language function via neurodegenerative diseases that target the left frontal or left anterior temporal lobes sometimes leads to the emergence of previously unrecognized visual or musical creativity" (p. 128). So, although some key aspects of left-brain function may be vital to a well-rounded creative process, it appears that an overly robust left brain can indeed get in the way.

Another popular theory in the neuroscience of creativity has focused on the role of the prefrontal cortex (PFC). Because the PFC is associated with planning, discernment, and rational decision-making, it seems a common-sense conclusion that any deliberate creative project would make extensive use of this area. The key word here, of course, is deliberate: many artists and even scientists report that creative breakthroughs happen outside of conscious deliberation. Arne Dietrich and his colleagues have done quite a bit of work to reconcile this contradiction by developing a neurobiological model that differentiates between deliberate creativity and spontaneous creativity (Dietrich \& Haider, 2017). Ultimately, both forms of creativity are vital and necessary, and they often have to work together, but Dietrich has been a staunch advocate for recognizing them as separate but interconnected processes in the brain. With regards to deliberate creative process, Dietrich (2004) points specifically to the dorsolateral prefrontal cortex (DLPFC), which appears to be the seat of working memory - the place where the brain holds relevant information while actively working on a project. At the same time, the actual workspace of working memory is limited, meaning that the conscious mind can only hold onto and work with so much information at a time. In order to keep working and looking for new ideas, images, and solutions, the working memory must access the sensory and association cortices, which operate outside the purview of direct conscious control, hence spontaneously. This is again an instance of the conscious mind turning toward the nonconscious processes of the brain for support. 
A similar neurobiological account of creativity was given by Damasio in 2001. Here he talks about the spontaneous generation of images (a term he uses somewhat synonymously with ideas), and the role that the prefrontal cortex plays in sorting through them:

The images are not realized as such in the prefrontal cortices-but rather in the early sensory cortices - but they are conducted or ordered from there ... I suspect that a marvelous prefrontal cortex generating many new items and holding them 'on line' would be of little use if we did not have the ability to execute good selections based on aesthetic or scientific goals. (p. 65)

The raw materials for creative work arise from the non-conscious activities of the sensory and association cortices, and they are then evaluated and worked over by the prefrontal cortex. Both aspects of this process are indispensable: without the PFC, the flow of images and ideas would be endless and amorphous. But without the contributions of the non-conscious brain regions, the PFC would have almost nothing to work with.

Dietrich and Haider (2017) further point out that the workspace of the PFC is constrained not only by limits on conscious processing-but also by biases and assumptions of all kinds. Specifically, the ventromedial prefrontal cortex is associated with managing emotional and social considerations and rules, and this factor can further shape and limit the kinds of ideas, images, and solutions that are deemed acceptable. We all have sets of beliefs and assumptions about what is real, what is possible, and what is acceptable, and these beliefs and assumptions can considerably limit any and all creative output.

Being at the top of the neural pyramid, the prefrontal cortex also houses a person's cultural values and belief system. This gives the deliberate mode - initiated and supervised by the prefrontal cortex as it is - a number of critical built-in predispositions as it generates new ideas . . . the deliberate mode is, for all its sightedness, pretty useless when the solution violates something we think is true about the world. (Dietrich, 2015, pp. 150-151)

Thus, a creative process that emphasizes the deliberate mode is useful for common-sense analytical solutions within the context of well-defined systems but not so good at major revelations, artistic breakthroughs, or new ways of looking at a problem. Dietrich and Haider (2017) ironically summarize: "While the deliberate mode has the advantage of limiting the solution space, it has the disadvantage of limiting the solution space!" (p. 5).

This constraint, of course, begins to push up against the very idea of creativity. If creative process is supposed to result in something novel and useful, the closed deliberate process of the PFC is only going to go so far. For a true experience of something wholly original emerging in the process of creative work, we must turn to regions of the brain that exist outside of conscious control.

\section{Neurobiology of the Creative Unconscious}

There is now a general consensus that the vast majority of what goes on in the brain does not reach conscious awareness. This notion is usually referred to with the 
technical term non-conscious processes, although occasionally a neuroscientist will be a little more poetic about it; Damasio, for example, writes that "there is a subterranean underneath the conscious mind and there are many levels to that subterranean" (1999, p. 319). If creativity is indeed a whole-brain process, and much of the brain is operating in this unconscious subterranean, it follows that a great deal of creativity must rely on the unconscious. Dietrich's (2004) model for deliberate creativity claims that "the working memory buffer of the prefrontal cortex holds the content of consciousness and that the attentional network of the prefrontal cortex is the mechanism to select and limit the content" (p. 1016). The content itself, however, is not being generated by the PFC per se but by the vast non-conscious processes of the brain. For this reason Damasio (2001) argues that creativity requires "strong generation of representational diversity ... the ability to generate - to bring to your conscious mind - a variety of novel combinations of entities and parts of entities as images" (p. 65). Furthermore, phenomenological inquires of all kinds have repeatedly reported that the creative contents generated by the unconscious are not always unformed chaos. On the contrary, human experience tells us that creative ideas and images can show up in varying degrees of complexity, from the chaotic and fragmentary to the sublime and whole (for examples and further discussion of this phenomenon, see Cameron, 1992; Csikszentmihalyi, 1996; Gilbert, 2015).

Several references have been made thus far to the association cortices, areas in the frontal, parietal, and temporal lobes that seem to be involved in connecting ideas. Pfenninger (2001) characterizes the association areas of the cortex as structures showing "extensive connections with other areas of the cerebral cortex and specialized for holding "mental images"' (p. 96). Andreasen (2005) further notes that the association cortices are marked by "a more complicated columnar organization than other parts of the brain" that are designed to connection information "in potentially novel ways" (p. 73). Although she does not reference the disinhibition hypothesis directly, she offers a similar theory in that, once the association cortices get going, they are no longer constrained in the way a common-sense thought process would require. These regions begin "running unchecked, not subject to any of the reality principles normally governing them" (p. 77). In theory, this process allows for ideas, images, and solutions that are outside of the ordinary, transcendent to limiting beliefs, and well beyond anything a deliberate conscious thought process is likely to come up with.

Perhaps the most sophisticated theory of unconscious association processes, as they relate to creative output, can be found in Gabora \& Ranjan's (2013) theory of neurds. This humorously titled term is used to refer to associative neural cliques within the vast networks of the brain. Their theory rests on the "distributed, content-addressable architecture of memory" (p. 22), which bears further explanation. The fact that memory appears to be distributed throughout the brain means that the various aspects of a given memory are actually spread and stored in different cortical regions. So, for example, the memory of a day at the beach with the family will have its visual components stored in the areas that process vision, the salty smell in the olfactory areas, the sounds of the waves in the temporal region, and so on. That memory is content-addressable means that ideas and images with similar features are stored together in associative neural cliques. In theory, it is only because memories are organized this way-with ideas and images stored in close association to other related ideas and images- that associative thinking is enabled 
to begin with. Thus, while each piece of memory has its own dedicated network of neurons, each of those neurons also branches out into the vast expanse of related images and ideas.

Gabora and Ranjan (2013) assert that in a linear, analytical thought process the brain follows established informational pathways to arrive a single piece of correct information. But in an associative thought process, it is not only the established connections of memory that are being accessed but also the wider network of associations attached to each piece of that memory. Neurds, then, are neural cliques "that would not be included in cell assembly if one were in an analytic mode of thought, but would be included if one were in the associative mode of thought" (p. 31). Such associations would only cloud and complicate a linear analytical thought process, but when analysis fails, the activation of the associative connections get the creative process going. "Their time to shine comes when one has to break out of a rut" (p. 31). For Gabora and Ranjan, this model settles the seemingly magical nature of creativity, whereby something emerges in the mind that did not exist previously.

Although the theory of neurds goes far in explaining how the brain recombines bits and pieces of information to make new ideas and images, it fails to address the extraordinary creativity seen in instances of artistic genius or scientific breakthrough. To remake a vision of the world requires more than putting a few ideas together in a new way. Andreason takes the discourse somewhat further in her discussion of complexity theory: "The human brain is perhaps the most superb example of a self-organizing system .... it is constantly and spontaneously generating new thoughts, often without external control" (2005, p. 63). Complex, self-organizing systems are identified by their uncanny ability to become measurably more than the sum of their parts (Waldrop, 1992). By applying this model, higher order creativity in the brain might be understood as an emergent process resulting from the ongoing and simultaneous intercommunication of multiple association areas on a number of different levels. Despite being scientifically measurable, complex systems retain an air of mystery and magic through this phenomenon of self-organizing emergence and as such may prove a more comfortable middle ground for those who do not want to abandon creativity completely to strict materialist models. Furthermore, the notion of a complex, self-organizing non-conscious brain takes us far toward in reconciling Jung's notion of an inherently creative unconscious.

The above theories of associative thinking in the brain can help us to understand how creative ideas are generated in the present moment, but they do less to explain the time-delayed creative breakthroughs that the classical model described in the cycle of incubation and illumination (or insight). Although criticism has been leveled at the notion that incubation and insight are always present in successful creative projects, the phenomenon is too widely reported to be entirely dismissed. Csikszentmihalyi's (1996) extensive study on highly creative individuals affirms incubation experiences as a common and important element for many who regularly practice creativity. Writing more recently, Kaufman and Gregoire (2015) argue that "research has supported the idea that the creative incubation that occurs during daydreaming is critical to creative thought and achievement and also to insightful problem solving" (p. 34). Dietrich (2015) too has emerged as a strong voice arguing for the plausibility of productive creative incubation: 
"the fact that groundbreaking discoveries in science are the result of the mind idling in neutral should give us pause the next time we want to credit creative thinking solely to the higher cognitive functions of the cerebral cortex." (p. 153). Such discussion gives more credence to the notion of an unconscious intelligence (as opposed to a merely nonconscious processes) if difficult problems are being worked out behind the scenes more or less all the time.

Dietrich and Haider (2017) have proposed a neurobiological account to help explain the incubation phenomenon. According to their theory, any active conscious processing on a project or problem carries with it a kind of neural inertia that continues even after the conscious mind has turned its attention elsewhere. "We can expect that a strongly interacting coalition of neurons does not instantly decay back to baseline. Any disintegration phase would take time, during which a new task set would be subjected to interference from the previous one" (p. 4). This would imply that a great deal of conscious focus on a problem would be a necessary precursor to incubation, as would repeated attempts to solve the problem. At best, however, this model functions as an explanation for incubation and insight in the medium-term only. It does not go far in explaining how the solution to a problem might emerge weeks or months after the project has been set aside.

\section{Conclusion}

Jung is sometimes criticized in more materialist circles for characterizing the unconscious as a source of intelligence or wisdom. But even on this issue there is evidence enough to at least consider what kinds of intelligence might exist beyond the conscious ego. Kaufman and Gregiore (2015) assert: "The brain's implicit processing system can, in fact, be very intelligent. Non-conscious processes may indeed be faster and structurally more sophisticated than our conscious thinking systems" (p. 66). Much research into this question remains to be done, and it is in the nature of such questions that they are extremely difficult to measure. Jung, of course, did not spend his life in the laboratory making measurements, but rather developed his theories through actual practice with living humans. His theories were born from clinical practice and introspection, and as such have a relational, imaginative, and sometimes outright poetic flavor that will never entirely fit with the dry technical language of the neurosciences.

Nevertheless, there is quite a bit in contemporary neuroscientific understanding to support Jung's ideas about creativity and, I would argue, vice versa. That there is a limit to what the analytical and deliberate processes of the conscious mind can achieve is quite similar to Jung's suggestion that intellect and conscious ego alone are not enough for creativity to occur. Likewise, though the terminology is different and the models more granular, the neurobiological recourse to non-conscious processes and associative thinking as a precursor to deliberate creativity is not unlike the lotus rising from the depths into conscious awareness. The latter just happens to be more poetic, and with good cause-for many people poetic, evocative language is far more resonant than the technical terminologies often employed in scientific discourse. Most importantly, the recognition by so many prominent neuroscientists that the conscious processes and unconscious processes of the brain must work together is itself a detailed neurobiological analogy for Jung's "mutual penetration of the conscious and unconscious" (1934/1954, p. 
152, para. 327). One does not have to believe that the brain is identical to the psyche to see meaningful and potentially fruitful parallels between the two fields

At the same time, we of the depth psychological persuasion might be mindful that we do not allow the current prominence of neuroscientific models to eclipse or, at worst, devour our field. Meditation worked for thousands of years before neuroscience validated it - it was an effective practice, long before it was objectively measured. So it is with depth psychology: we can celebrate communion with the neurosciences without dissolving and reducing the practices and understandings of depth psychology to neurobiological accounts. In the end, the psyche is not the brain; it is the psyche, a map all its own. As such, creativity can indeed be considered a birthright, and not just because many neuroscientists concur that it is so. It is a birthright simply because throughout the long stretch of human existence, from the first cave paintings and stories told by firelight, creativity has been a source of meaning and joy. It is part and parcel of who we are, something to be experienced and appreciated and known from within.

\section{Contributor}

Jonathan Erickson, Ph.D., is a writer and life coach based in Portland Oregon. He completed his doctorate in depth psychology and somatic studies from Pacifica Graduate Institute, where his dissertation research focused on neuroscience and imagination. His previous publications include papers on psychology and religion, human-animal relationships, and virtual embodiment in video games.

\section{References}

Andreasen, N. (2005). The creative brain: The science of genius. New York, NY: Plume. Cameron, J. (1992). The complete artist's way. New York, NY: Jeremy P. Tarcher/Penguin.

Carson, S. (2010). Your creative brain. San Francisco, CA: Jossey-Bass.

Csikszentmihalyi, M. (1996). Creativity: Flow and the psychology of discovery \& invention. New York, NY: HarperCollins Publishers.

Damasio, A. (1999). The feeling of what happens. New York, NY: Harcourt.

Damasio, A. (2001). Some notes on brain, imagination, and creativity. In K. H. Pfenninger, \& V. Shubik (Eds.), The origins of creativity (pp. 89-97). New York, NY: Oxford University Press.

Dietrich, A. (2004). The cognitive neuroscience of creativity. Psychonomic Bulletin and Review, 11(6), 1011-1026. doi:10.3758/BF03196731

Dietrich, A. (2007). Who is afraid of a cognitive neuroscience of creativity? Methods 42, 22-27. doi:10.1016/j.ymeth.2006.12.009

Dietrich, A. (2015). How creativity happens in the brain. New York, NY: Palgrave Macmillan.

Dietrich, A. \& Haider, H. (2017). A neurocognitive framework for human creative thought. Frontiers in Psychology 7(2078), 1-7. doi:10.3389/fpsyg.2016.02078 
Gabora, L., \& Ranjan, A. (2013). How insight emerges in a distributed, contentaddressable memory. In O. Vartanian, S. Bristil, \& J. C. Kaufman (Eds.), Neuroscience of creativity (pp. 19-44). Cambridge, MA: MIT Press.

Gilbert, E. (2015). Big magic: Creative living beyond fear. New York, NY: Riverhead Books.

Helmholtz, H. V. (1896). Vorträge und reden. Brunswick, Germany: Friedrich Viewig und Sohn.

Jung, C. G. (1954). The practical use of dream-analysis (R. F. C. Hull, Trans.). In H. Read et al. (Series Eds.), The collected works of C. G. Jung (Vol.16, 2nd ed., pp. 139-162). New York, NY: Princeton University Press. (Original work published 1934)

Jung, C. G. (1954). The psychology of the transference (R. F. C. Hull, Trans.). In H. Read et al. (Series Eds.), The collected works of C. G. Jung (Vol.16, 2nd ed., pp. 163-202). Princeton, NJ: Princeton University Press. (Original work published 1946)

Jung, C. G. (1969). The transcendent function (R. F. C. Hull, Trans.). In H. Read et al. (Series Eds.), The collected works of C. G. Jung (Vol.8, 2nd ed., pp. 67-91). Princeton, NJ: Princeton University Press. (Original work published 1957)

Jung, C. G. (1971a). The problem of types in the history of classical and medieval thought (R. F. C. Hull, Trans.). In H. Read et al. (Series Eds.), The collected works of C. G. Jung (Vol. 6, pp. 8-66). Princeton, NJ: Princeton University Press. (Original Published in 1921)

Jung, C. G. (1971b). Schiller's ideas on the type problem (R. F. C. Hull, Trans.). In H. Read, et al. (Series Eds.), The collected works of C. G. Jung (Vol. 6, pp. 67-135). Princeton, NJ: Princeton University Press. (Original Published in 1921)

Jung, C. (1976). Symbols and the interpretation of dreams (R. F. C. Hull, Trans.). In H. Read et el. (Series Eds.), The collected works of C. G. Jung (Vol.18, pp. 183-264). New York, NY: Princeton University Press. (Original work published 1961)

Jung, R. E., \& Haier, R. J. (2013). Creativity and intelligence: Brain networks that link and differentiate the expression of genius. In O. Vartanian, S. Bristil, \& J. C. Kaufman (Eds.), Neuroscience of creativity (pp. 233-254). Cambridge, MA: MIT Press.

Kaufman, A. B., Kornilov, S. A., Bristol, A. S., Tan, M., \& Grigorenko, E. L. (2010). The neurobiological foundation for creative cognition. In J. C. Kaufman \& R. J. Sternberg (Eds). The Cambridge handbook of creativity (pp. 216-232). New York, NY: Cambridge University Press.

Kaufman, J. C., \& Beghetto, R. A. (2009). Beyond big and little: The four-C model of creativity. Review of General Psychology 13(1), 1-12.

Kaufman, S. B. \& Gregoire, C. (2015). Wired to create: Unraveling the mysteries of the creative mind. New York, NY: Tarcher Perigee.

McGilchrist, I. (2009). The master and his emissary: The divided brain and the making of the Western world. New Haven, CT: Yale University Press. 
Pfenninger, K. H. (2001). The evolving brain. In K. H. Pfenninger, \& V. Shubik (Eds.), The origins of creativity (pp. 89-97). New York, NY: Oxford University Press.

Radel, R., Davranche, K., Fournier, M., Dietrich, A. (2015). The role of (dis)inhibition in creativity: Decreased inhibition improves idea generation. Cognition 134, 110120 .

Sperry, R. W. (1974). Lateral specialization in the surgically separated hemispheres. In F. Schmitt \& F. Worden (Eds.), Third neurosciences study program (Vol. 3, pp. 519). Cambridge, MA: MIT Press.

Vartanian, O., Bristil, S., \& Kaufman, J. C. (2013). Neuroscience of creativity. Cambridge, MA: MIT Press.

Vishkontas, V. \& Miller, B. L. (2013). Art and dementia: How degeneration of some brain regions can lead to new creative impulses. In O. Vartanian, S. Bristil \& J.C. Kaufman (Eds.) Neuroscience of creativity. Cambridge, MA: MIT Press.

Waldrop, M. (1992). Complexity: The emerging science at the edge of order and chaos. New York, NY: Simon and Schuster.

Wallas, G. (1926). The art of thought. New York, NY: Harcourt Brace 